\title{
A pesquisa translacional e o cuidado perineal no parto: relato de implementação
}

\section{Rafael Cleison Silva dos Santos ${ }^{1}$, José Lucio Dantas Júnior Farias ${ }^{2}$ e Clodoaldo Tentes Côrtes ${ }^{3}$}

\footnotetext{
1 Doutor em Ciências do Cuidado de Saúde pela Universidade de São Paulo. Professor Adjunto do Departamento de Ciências Biológicas e da Saúde da Universidade Federal do Amapá, Brasil.

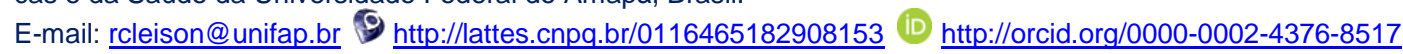

2 Especialista em Enfermagem em Obstetrícia pelo Programa de Residência em Enfermagem da Secretaria de Estado da Saúde do Amapá. Enfermeiro no Instituto Federal do Amapá, Brasil.

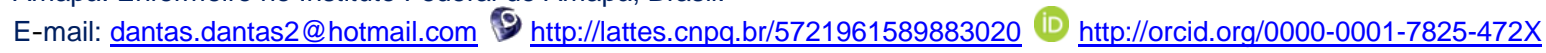

3 Doutor em Ciências do Cuidado de Saúde pela Universidade de São Paulo (USP). Professor Adjunto do Departamento de Ciências Biológicas e da Saúde da Universidade Federal do Amapá, Brasil.

E-mail: ctcortes@unifap.br (9) http://lattes.cnpq.br/9489426188166592 iD http://orcid.org/0000-0002-8512-4250
}

RESUMO: A pesquisa translacional é uma metodologia que tem sido proposta para a transferência de conhecimento com a finalidade de melhorar a qualidade do cuidado de saúde. O cuidado no parto inclui a prevenção, classificação e reparo do trauma perineal baseado nas meIhores evidências científicas disponíveis. O objetivo foi descrever os aspectos operacionais da metodologia de implementação de evidências utilizada em uma pesquisa translacional para prevenção e reparo do trauma perineal no parto. Trata-se de relato de experiência sobre a utilização da metodologia do Instituto Joanna Briggs, Austrália, aplicada em uma maternidade pública do Estado do Amapá. As seguintes atividades foram realizadas: constituição da equipe de auditoria, definição dos critérios e auditoria de base; implementação das melhores práticas; auditoria pós-implementação. Conclui-se que apesar das barreiras percebidas na pesquisa, alguns facilitadores foram observados no local do estudo, após a implementação das práticas baseadas em evidências científicas.

Palavras-chave: Auditoria clínica. Parto. Períneo. Pesquisa translacional. Prática clínica baseada em evidências.

\section{Translational research and perineal care at childbirth: implementation report}

ABSTRACT: Translational research is a methodology that has been proposed for the transfer of knowledge in order to improve the quality of health care. Childbirth care includes the prevention, classification, and repair of perineal trauma based on the best available scientific evidence. The objective was to describe the operational aspects of the methodology of evidence implementation used in a translational research to prevent and repair perineal trauma at childbirth. This is an experience report about the use of the methodology of the Joanna Briggs Institute, Australia, applied in a public maternity of the state of Amapá. The following activities were carried out: constitution of the audit team, definition of the criteria and basic audit; Implementation of best practices; Post-implementation audit. It is concluded that despite the perceived barriers in the research, some facilitators were observed at the study site, after implementation of practices based on scientific evidence.

Keywords: Clinical audit. Childbirth. Perineum. Translational research. Evidence-based clinical practice. 


\section{INTRODUÇÃO}

No presente trabalho apresenta-se o relato de experiência sobre uma pesquisa translacional realizada no Estado do Amapá para implementar evidências científicas na prática clínica relacionada à prevenção e ao reparo do trauma perineal no parto normal.

A pesquisa translacional é um termo da língua inglesa, translational research, mas que tem sido usado na língua portuguesa como sinônimo de transferência de conhecimento, ciência translacional ou ciência da tradução ou tradução do conhecimento (CÔRTES et al., 2015; SANTOS; RIESCO, 2016).

Apesar das dificuldades de traduzir o conhecimento e implementar práticas baseadas em evidências, esse tipo de experiência reduz a lacuna entre a investigação e a prática clínica, através da divulgação e da troca de evidências de pesquisas e sua aplicação à prática clínica para melhorar os resultados da saúde, a qualidade dos cuidados e dos sistemas de cuidados de saúde (STEVENS et al., 2014; JORDAN et al., 2016).

Em relação ao trauma perineal, este pode ocorrer espontaneamente (laceração) durante o parto vaginal ou como consequência de uma incisão cirúrgica (episiotomia) para ampliar a abertura vaginal. 0 trauma pode ser classificado em laceração de primeiro grau (lesão da pele ou mucosa vulvo-vaginal), segundo grau (secciona a musculatora), terceiro grau (envolve os esfincteres) até uma laceração de quarto grau, que é a mais grave lesão perineal e envolve o epitélio anal (CARROLI; MIGNINI, 2012).

Sobre o cuidado perineal, poucos estudos sobre a prevenção do trauma perineal no parto vem sendo realizados do mundo.
No entanto, é sabido que prevenir o trauma perineal no parto tem impacto contra as principais morbidades decorrentes, especialmente, sangramento, dor, infecção, deiscência e dispareunia (AMERICAN COLLEGE OF OBSTETRICIANS AND GYNECOLOGISTS, 2016). Além disso, pesquisas mostram que a maioria das mulheres que tem parto vaginal sofre algum grau de trauma perineal e que mais da metade necessita de reparo para facilitar a cicatrização do tecido lesado (BICK et al., 2012; ISMAIL et al., 2013; KETTLE; DOWSWELL, ISMAIL, 2013)

O objetivo deste trabalho foi descrever os aspectos operacionais da metodologia de implementação de evidências, utilizada em uma pesquisa translacional para prevenção e reparo do trauma perineal no parto.

\section{MÉTODO}

Trata-se de um relato de experiência profissional, vivenciada pelos autores durante uma pesquisa translacional para implementar evidências científicas na prevenção e reparo do trauma perineal no parto.

A referida pesquisa consistiu em um estudo de intervenção quase experimental, tipo antes e depois, segundo a metodologia de implementação de evidências científicas na prática clínica do Instituto Joanna Briggs (JBI), Austrália, que possui três fases (préauditoria e auditoria de base, implementação das melhores práticas e auditoria pósimplementação). Essa metodologia facilita o processo de mudança pela auditoria clínica, identificação do problema, planejamento de ação, implementação de ações e reauditoria, como estratégias para melhorar a adoção das melhores evidências disponíveis sobre um tema de interesse (PEARSON; JORDAN; MUNN, 2012; JORDAN et al., 
2016).

O local do estudo foi em um centro de parto normal de uma maternidade pública de referência em Macapá, Amapá, entre julho de 2015 e março de 2016, envolvendo enfermeiros, médicos, puérperas e prontuários. Em 2016, segundo o Serviço de Arquivo Médico e Estatística, a média mensal de partos no local do estudo foi de 650 , com $67 \%$ de partos normais.

A pesquisa foi aprovada pelo Comitê de Ética em Pesquisa da Escola de Enfermagem da Universidade de São Paulo, protocolo CAAE: 31700414.50000 .5392 . A participação dos profissionais e puérperas foi voluntária, após a leitura e assinatura do Termo de Consentimento Livre e Esclarecido e Termo de Assentimento, quando indicado.

\section{A EXPERIÊNCIA DA PESQUISA}

$\mathrm{Na}$ experiência de utilizar uma metodologia de transferência de conhecimentos baseados em evidências científicas para a implementação de cuidados perineais no parto normal, destacam-se as seguintes atividades: a) constituição da equipe de auditoria, definição dos critérios de auditoria (pré-auditoria) e avaliação preliminar dos desfechos e práticas utilizadas na prevenção e reparo do trauma perineal (auditoria de base); b) intervenção educativa para implementação das melhores práticas; c) auditoria pós-implementação.

A equipe de auditoria foi constituída pelos diretores, coordenadores da pesquisa e um enfermeiro residente. Além disso, os seguintes critérios de auditoria foram estabelecidos: posição no parto; puxos dirigidos; manobra de Kristeller, prevenção do trauma perineal; taxas de episiotomia e laceração espontânea; avaliação e classifica- ção da laceração; reparo de laceração de primeiro grau; técnica de sutura do trauma perineal; tipo de fio; anestesia no reparo perineal; dor perineal e satisfação da puérpera com o reparo perineal.

Para avaliar os desfechos maternos e identificar as práticas utilizadas no local do estudo, antes da intervenção educativa, realizaram-se 42 entrevistas face-a-face com profissionais (17 médicos e 25 enfermeiros), especialistas em obstetrícia e que atuavam na assistência ao parto e outras 35 entrevistas face-a-face com mulheres não indígenas e de risco habitual obstétrico (entre 1-2 dias de internação pós-parto, no hospital), e por telefone, após a alta (entre 10-12 dias e 30 dias pós-parto). Além disso, coletou-se dados em 291 prontuários de puérperas não entrevistadas, considerando os mesmos critérios de inclusão das puérperas entrevistadas.

As perguntas aos profissionais relacionavam-se as práticas na assistência ao parto, quais e com que frequência o profissional as realizava: a posição que recomendavam no período expulsivo do parto, o tipo de puxo, a técnica de prevenção do trauma, a prática de episiotomia, a classificação e o reparo do trauma, a realização do exame retal depois do reparo de lacerações de 3 ㅇ grau, a técnica de reparo perineal, e o material do fio de sutura utilizado.

Em relação às mulheres, as perguntas diziam respeito às práticas vivenciadas no parto, o cuidado perineal que a puérpera recebeu, e como elas se sentiram depois da alta hospitalar: a posição que ficou na hora do parto, se mandaram fazer força para baixo no período expulsivo (puxo dirigido), se fizeram força no fundo da barriga (manobra de Kristeller), se cortaram (episiotomia) ou se rasgou a vagina (laceração), se 
fizeram anestesia antes do corte ou antes dos pontos, se foi feito pontos, caso tenha ocorrido o rasgo, as morbidades apresentadas nos pontos, se tomou alguma medicação para dor nos pontos, e o grau de satisfação em relação aos pontos na vagina.

A fase de implementação das melhores práticas foi desenvolvida mediante três intervenções educativas para os profissionais (dois seminários e um banner). Os seminários, realizados um pela manhã e outro à tarde, três semanas após o término da auditoria de base, tiveram quatro horas de duração cada, para possibilitar a participação de todos os profissionais envolvidos. A finalidade foi apresentar os resultados da avaliação preliminar das práticas utilizadas na maternidade, discutir as evidências científicas disponíveis na literatura e buscar estratégias de mudança na prática desses profissionais. Além disso, foi disponibilizado material de leitura atualizado com tradução para o português (ensaios clínicos e revisões sistemáticas) e certificação para cada profissional.

O banner, que media $120 \times 90 \mathrm{~cm}$ e continha a síntese das evidências apresentadas nos seminários, foi apresentado durante os 40 dias que sucederam o término dos seminários e discutido face-a-face pelos autores da pesquisa com profissionais que estiveram ausentes nos seminários. Ao fim do seminário, o banner foi disponibilizado para a educação continuada da instituição. No total, 32 profissionais participaram das três intervenções.

A fase de auditoria pós-implementação foi iniciada dois meses após a realização dos seminários e foi desenvolvida do mesmo modo que a auditoria de base, pelos mesmos pesquisadores e utilizando-se os mesmos instrumentos, com a finalidade de ava- liar o impacto das intervenções educativas. Nesta fase, os pesquisadores entrevistaram os 32 profissionais (20 enfermeiros e 12 médicos) que participaram das intervenções educativas e outras 35 puérperas, diferentes daquelas entrevistadas na primeira fase. Novos dados também foram coletados de 264 prontuários de puérperas não entrevistadas.

\section{DISCUSSÃO}

No presente trabalho, embora não seja objetivo discutir os achados da pesquisa, mas apenas relatar a experiência dos participantes, vale destacar que a intervenção educativa melhorou os cuidados e os desfechos perineais, incluindo menos profissionais enfermeiros e médicos que realizam puxos dirigidos e episiotomia de rotina e mais registros nos prontuários do uso do fio de sutura ácido poliglicólico ou poliglactina 910 na mucosa e na pele. Esses achados corroboram com o entendimento de que após a intervenção educativa, houve meIhora para essas práticas, com base nas evidências científicas disponíveis (KETTLE; DOWSWELL, ISMAIL, 2010; AMERICAN COLLEGE OF OBSTETRICIANS AND GYNECOLOGISTS, 2016; BRASIL, 2016).

Por outro lado, a pesquisa identificou lacunas na implementação de evidências e algumas inadequações no manejo do cuidado perineal, tais como, relatos de puérperas submetidas à posição de litotomia e falta de registros nos prontuários em relação à sutura das lacerações perineais. Em concordância com os achados do Inquérito Nacional Nascer no Brasil, nossa realidade é semelhante ao restante do país, com intervenções obstétricas durante o trabalho de parto e parto em mulheres de risco habitual 
em que os profissionais não sabem justificar cientificamente a sua prática (LEAL et al., 2014).

Sobre a translação de conhecimento, há pouca experiência disponível na literatura, principalmente para o cuidado perineal no parto normal. No entanto, nos últimos doze anos, sete estudos, descritos a seguir, incluindo três no Brasil, testaram essa metodologia de trabalho envolvendo o manejo do cuidado perineal.

A primeira pesquisa translacional apresenta um estudo realizado no Reino Unido com 208 profissionais (obstetrizes e médicos) que participaram de um workshop, dos quais, $71 \%$ responderam um questionário antes e oito semanas após o curso, mostrando que $64 \%$ estavam insatisfeitos com a sua formação antes de realizar seu primeiro reparo perineal sem supervisão. Após o workshop, houve aumento na capacidade dos participantes para classificar as lesões obstétricas do esfíncter anal com precisão. Houve aumento proporcional na prática de realização de exame retal, antes e depois de reparar o trauma perineal - $28 \%$ para $89 \%$. Significativamente, mais participantes mudaram para cuidados baseados em evidências, em especial, na técnica contínua de reparo perineal (ANDREWS et al., 2005).

A segunda experiência encontrada foi realizada em quatro países asiáticos (Tailândia, Indonésia, Filipinas e Malásia). Nesse estudo, foi analisada a prática de episiotomia, antes e depois de uma intervenção multicomponente, para apoiar o uso e a geração de evidências científicas na área da saúde materna. Na primeira fase (préintervenção), os dados foram obtidos por auditoria de práticas recomendadas (incluindo episiotomia seletiva) e resultados de saúde na gravidez e no parto (incluindo o períneo íntegro) e pela análise do conhecimento pessoal da prática baseada em evidências. A segunda fase incluiu as intervenções educativas baseadas em evidências (oficinas interativas e exposições de pôsteres). Na terceira fase, a auditoria foi repetida. Houve quedas na taxa de episiotomia e do trauma grave nas nulíparas (92,2\% para $80,7 \%$ e $6,7 \%$ para $3,0 \%$, respectivamente); além de aumento da frequência de períneo íntegro em nulíparas ( $1,7 \%$ para $8,0 \%$ ). Os autores concluíram que uma intervenção educativa baseada na utilização de evidências científicas resulta em melhorias na prática da episiotomia e na redução da taxa de trauma perineal entre as mulheres nulíparas (HO et al., 2010).

A terceira pesquisa foi realizada na cidade de São Paulo, Brasil, para verificar que as barreiras na transferência do conhecimento sobre o uso da episiotomia de rotina entre 63 profissionais (enfermeiras, obstetrizes e médicos) de três maternidades públicas eram a hesitação em aceitar a generalização da evidência, o papel do contexto na prática clínica e as atitudes e crenças sobre a importância da episiotomia no parto. Embora a quase totalidade dos participantes manifestasse opinião positiva em relação à implementação do cuidado baseado em evidências, os achados reafirmaram que, na prática, o comportamento é formado muito mais por crenças, atitudes e experiência que pelas evidências científicas. $O$ estudo também explorou a perspectiva de 217 puérperas sobre o uso da episiotomia, incluindo suas experiências na tomada de decisão sobre o cuidado perineal no parto. A maioria $(72,4 \%)$ afirmou não ter recebido informação sobre os motivos da realização da episiotomia; entretanto, muitas mulheres acreditavam que essa conduta era ne- 
cessária e que faz parte do cuidado no parto normal, indicando a falta de conhecimento sobre os riscos e benefícios da episiotomia (BASTOS, 2011).

Em 2012, foi publicado o quarto trabaIho, desta vez, realizado com 405 obstetrizes, no Reino Unido. Identificou-se como essas obstetrizes avaliavam e reparavam trauma perineal e a extensão em que a prática refletia as orientações baseadas em evidências. $O$ resultado mostrou que poucas obstetrizes (6\%) utilizavam métodos de sutura baseados em evidências, e que apenas $17,3 \%$ realizavam o exame retal como parte da rotina de avaliação do trauma e que mais da metade $(58,0 \%)$ não fazia sutura em todas as lacerações de segundo grau. Além disso, as obstetrizes com mais experiência profissional eram mais propensas a implementar as evidências. Contudo, os autores concluíram que há lacunas consideráveis em relação à implementação de evidências na prática para apoiar o manejo do trauma perineal relacionado ao parto (BICK et al., 2012).

Ainda no Reino Unido, identificou-se uma quinta pesquisa translacional, onde os autores realizaram um estudo pioneiro para avaliar a intervenção de um módulo de educação multiprofissional, destinado a melhorar os conhecimentos e as habilidades clínicas de obstetrizes e médicos para implementar o manejo e a avaliação de episiotomia e lacerações perineais de segundo grau, com base em evidências. Para tanto, foi desenvolvida uma metodologia de transferência de conhecimento, mediante um ensaio clínico randomizado em grupos pareados, com 22 maternidades e 3.681 mulheres. A pesquisa foi feita em diversas etapas, que incluíram a identificação dos desfechos perineais considerados importantes pelas mu-
Iheres, intervenção educacional com obstetrizes e médicos e comparação dos resultados intra e intergrupos, antes e depois da intervenção. Os resultados mostraram que a intervenção educacional melhorou o conhecimento dos profissionais sobre as práticas baseadas em evidências no manejo clínico do trauma perineal e favoreceu alguns desfechos clínicos maternos. Os autores concluíram que a educação permanente e padronizada é essencial para manutenção dessa melhoria no cuidado (ISMAIL et al., 2013).

No Brasil, em 2015, foi realizada a sexta pesquisa translacional em um centro de parto normal de uma maternidade municipal, na Região Metropolitana de São Paulo, Brasil, que utilizou o método de implementação de evidências científicas na prática clínica do JBI. O resultado da auditoria pósimplementação mostrou que a pesquisa trouxe impactos positivos, pois melhoraram os desfechos perineais no parto normal com redução da dor nos pontos da sutura perineal ao andar ou sentar, aumentou a satisfação das puérperas com o períneo e o número de profissionais que utilizou cuidados baseados em evidências (CÔRTES et al. 2015).

Em 2016, no Brasil, também foi publicada uma sétima pesquisa translacional realizada em um hospital público de referência no Estado do Amapá. Após a intervenção educativa, os resultados mostraram que a intervenção educativa melhorou os cuidados e os desfechos perineais, pois menos profissionais realizaram puxos dirigidos ou episiotomia, e os registros nos prontuários indicaram maior uso de fio sintético absorvível na sutura da mucosa e pele; porém, há lacunas na implementação das evidências e inadequações no manejo do cuidado perineal, 
uma vez que mais mulheres informaram que o parto foi em posição litotômica (SANTOS; RIESCO, 2016).

\section{CONCLUSÃO}

A experiência em participar desse projeto de implementação nos permitiu apontar algumas limitações e implicações deste método para a prática e para a pesquisa. As principais limitações foram a elevada falta de registros adequados das práticas nos prontuários e as dificuldades na coleta de dados e na participação de alguns profissionais, principalmente, os médicos. Sobre as implicações para a prática, este relato pode contribuir para o conhecimento dos profissionais que atuam na assistência ao parto, mostrando que muitos cuidados são empíricos e que existem evidências que podem prevenir muitas morbidades. Para a pesquisa, o relato mostrou que apesar das barreiras e dificuldades desta metodologia, as auditorias permitem a identificação oportuna de potenciais falhas no cuidado, de modo que estratégias podem ser estabelecidas para melhorá-lo.

\section{REFERÊNCIAS}

AMERICAN COLLEGE OF OBSTETRICIANS AND GYNECOLOGISTS. Prevention and management of obstetric lacerations at vaginal delivery. Practice Bulletin №. 165. Obstet Gynecol, v.128, n.15, 2016.

ANDREWS, V.; et al. Can hands-on perineal repair courses affect clinical practice? British journal of midwifery, v.13, n.9, p.562566, 2005. https://doi.org/10.12968/bjom. 2005.13.9.19625

BASTOS, M. H. S. Developing and pilottesting an educational intervention to re- duce the routine performance of episiotomy in Brazil. 2011. 293f. [thesis]. London: Thames Valley University, 2011.

BICK, D.; et al. How good are we at implementing evidence to support the management of birth related perineal trauma? A UK wide survey of midwifery practice. BMC Pregnancy and Childbirth, v.12,n.57, 2012. BRASIL. Ministério da Saúde; Secretaria de Políticas de Saúde, Área Técnica de Saúde da Mulher. Diretriz nacional de assistência ao parto normal: relatório de recomendação. Brasília, 2016.

CARROLI, G.; MIGNINI, L. Episiotomy for vaginal birth. Cochrane Database of Systematic Reviews, Issue 1. Oxford, 2012.

CÔRTES, C. T.; et al. Implementation methodology of practices based on scientific evidence for assistance in natural delivery: a pilot study. Rev Esc Enferm USP, v.49, n.5,p.716-724, 2015. https://doi.org/10. 1590/S0080-623420150000500002.

$\mathrm{HO}, \mathrm{J} . \mathrm{J}$.; et al. Influence of training in the use and generation of evidence on episiotomy practice and perineal trauma. International Journal of Gynecology and Obstetrics, v.111, p.13-18, 2010. https://doi.org/ 10.1016/i.ijgo.2010.04.035

ISMAIL, K. M.; et al. Perineal Assessment and Repair Longitudinal Study (PEARLS): a matched-pair cluster randomized trial. BMC Medicine, v,11, n.209, 2013. https://doi. org/10.1186/1741-7015-11-209.

JORDAN, Z. The updated JBI model for evidence-based healthcare. The Joanna Briggs Institute; 2016.

KETTLE, C.; DOWSWELL, T.; ISMAIL, K. M. Continuous and interrupted suturing techniques for repair of episiotomy or seconddegree tears. Cochrane Database of Systematic Reviews, Issue 11, 2013. https:// doi.org/10.1002/14651858.CD000947.pub3. 
KETTLE, C.; DOWSWELL, T.; ISMAIL, K. M. Absorbable suture materials for primary repair of episiotomy and second degree tears. Cochrane Database of Systematic Reviews, Issue 6, 2010. https://doi.org/10. 1002/14651858.CD000006.pub2.

LEAL, M. C.; et al. Obstetric interventions during labour and birth in Brazilian low risk women. Cad Saúde Public, v.30, n.4, 2014.

PEARSON, A.; JORDAN, Z.; MUNN, Z. Translational science and evidence-based healthcare: a clarification and reconceptualization of how knowledge is generated and used in healthcare. Nurs Res Pract, 2012. https:// doi.org/10.1155/2012/792519

SANTOS, R. C. S.; RIESCO, M. L. G. Implementação de práticas assistenciais para prevenção e reparo do trauma perineal no parto. Rev Gaúcha Enferm, v.37, n.(esp), 2016. http://dx.doi.org/10.1590/1983-144 7.2016.esp.68304.

STEVENS, B. J.; et al. Pain in hospitalized children: Effect of a multidimensional Knowledge translation strategy on pain process and clinical outcomes. Pain, n.155, p.60-68, 2014. https://doi.org/10.1016/i. pain.2013.09.007

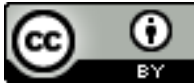

License information: This is an openaccess article distributed under the terms of the Creative Commons Attribution License, which permits unrestricted use, distribution, and reproduction in any medium, provided the original work is properly cited.

Artigo recebido em 11 de agosto de 2017.

Avaliado em 29 de junho de 2018.

Aceito em 02 de julho de 2018.

Publicado em 28 de fevereiro de 2019.
Como citar este artigo (ABNT):

SANTOS, Rafael Cleison Silva dos; FARIAS, José Lucio Dantas Júnior; CÔRTES, Clodoaldo Tentes. A pesquisa translacional e o cuidado perineal no parto: relato de implementação. Estação Científica (UNIFAP), Macapá, v. 8, n. 2, p. 121-128, maio/ago. 2018. 